\title{
Diseño e implementación de una práctica de metacognición en la asignatura de Fisiología Humana (Facultad de Medicina)
}

\author{
J.F. Escanero-Marcen, C. González-Haro, L. Ezquerra, L. Borque
}

Objetivo. Presentar el diseño y los resultados obtenidos en una práctica sobre metacognición en Fisiología Humana (Facultad de Medicina). Sujetos y métodos. Ciento diecinueve estudiantes (95 mujeres y 24 varones) asistieron a una práctica sobre metacognición en la asignatura de Fisiología Humana (Facultad de Medicina). La práctica se dividió en dos partes: en la primera, los estudiantes se determinaron el estilo de aprendizaje (test de Kolb) para poner de manifiesto que no todos aprenden de la misma manera, y se les hizo reflexionar sobre las características esenciales de cada estilo encontrado; en la segunda, escribieron las recomendaciones que harían a su mejor amigo/a sobre cómo estudiar fisiología y, posteriormente, las expusieron ante el resto. Resultados y conclusiones. Los resultados respecto a los modos de aprender mostraron dos hechos: uno, que la mayor puntuación se obtuvo en la conceptualización abstracta, tanto para varones como para mujeres, y, otro, referente a la segunda opción, que las mujeres señalaron la experimentación activa (significativamente mayor que en los varones), mientras que para éstos fue la observación reflexiva. Los estilos de aprendizaje de los estudiantes fueron convergentes y asimiladores, con escasa diferencia entre ambos. Por sexos, se observó que en los varones predominó el estilo asimilador (13 frente a 8), mientras que en las mujeres fue más frecuente el convergente (44 frente a 36). En la segunda parte de la práctica (metacognición) ambos grupos manifestaron utilizar las mismas estrategias, con independencia del estilo al que pertenecían. Determinadas estrategias fueron utilizadas por los estudiantes con niveles de sofisticación diferentes.

Palabras clave. Estilos de aprendizaje. Estrategias de aprendizaje. Metacognición.

\begin{abstract}
Designing and implementing a practical on metacognition as part of the subject of Human Physiology (Faculty of Medicine)
\end{abstract}

Aim. To present the design and results found in a practical lecture on metacognition in the subject of Human Physiology (School of Medicine). Subjects and methods. The practical lecture was attended by 119 students $(95$ women and 24 men). This one was divided into two parts: in the first, students determined by themselves their learning style (Kolb test) in order to show that not everyone learns the same way and makes them reflect on the essential features of each style found; in the second, they wrote on the recommendations made to his/ her best friend on how to study the Physiology and then put them to rest. Results and conclusions. The results respect to the learning modes showed two facts: one, the highest score was obtained by abstract conceptualization, for both men and women; and another, in relation to the second option the active experimentation was reported by women (significantly greater than men) versus reflective observation indicated by men. Regarding learning styles of students, these were assimilator and converger, with little difference between them. By gender, the following fact was found: in men predominated assimilator style (13 versus 8), whereas women were the most converger one (44 versus 36). In the second part of the practical lecture (metacognition) both groups expressed to use the samelearning strategies, independently of the learning style. Some strategies were used by students with different sophistication levels.

Key words. Learning styles. Learning strategies. Metacognition.

\section{Introducción}

Tradicionalmente, la enseñanza en la universidad se ha centrado en la transmisión de los
Departamento de

Farmacología y Fisiología. Facultad de Medicina. Universidad de Zaragoza. Zaragoza, España.

Correspondencia Dr. Jesús F. Escanero Marcen. Departamento de Farmacología y Fisiología. Facultad de Medicina. Universidad de Zaragoza. Domingo Miral, s/n. E-50009 Zaragoza.

E-mail escanero@unizar.es 
conocimientos relativos a una determinada materia y a su aprendizaje memorístico. En la actualidad, se ha puesto de manifiesto que el tipo de conocimientos que responde 'a lo que hay que aprender' es restrictivo y que existen otros igual de importantes. Se refiere a los conocimientos sobre el cómo -cognitivos y metacognitivos- y el porqué -conocimientos condicionales- [1]. En esta línea, Monereo y Pozo [2] señalan que en la universidad se ha considerado que lo primero y principal consiste en adquirir los conocimientos básicos que posteriormente permitirán pensar sobre ellos para poder utilizarlos un día de forma reflexiva y resolutiva; explican además que algunos autores han propuesto, de forma acertada, trastocar esta norma y pensar sobre los contenidos que se aprenden mientras se aprenden, de forma que aprender sea una consecuencia de pensar. 'La reflexión debería ser el centro de las actividades de aprendizaje que se producen en el aula (presencial o virtual), dejando la transmisión de la información pura y dura en un segundo plano o restringida a sistemas automatizados de búsqueda y navegación en redes telemáticas' [2].

Por otra parte, Pérez-Echeverría et al [3], compararon el aprendizaje entre expertos y novatos (con problemas de física, de álgebra y con tareas de salud y enfermedad) y sugirieron que los novatos en diferentes áreas organizan sus conocimientos de forma distinta a los expertos. Estos últimos organizan sus conocimientos a partir de leyes o principios generales que dan sentido a los problemas prácticos o reales, mientras que los novatos los organizan a partir de los propios escenarios o situaciones a los que se enfrentan.

En la actualidad, como indican FernándezBorrás et al [4], se ha sugerido que las estrategias de aprendizaje se ubican en el mismo nivel jerárquico que los conocimientos temáticos específicos de cada disciplina. Desde este punto de vista, el conocimiento de las mismas se muestra como una acción prevalente en las instituciones dedicadas a la enseñanza superior. A este respecto, Coffield et al [5] escriben: 'Los aprendedores llegan a ser más eficaces como aprendedores si ellos son conscientes de las importantes cualidades que ellos y otros aprendedores poseen'.

Teniendo en cuenta todo lo anterior, y con el fin de que los estudiantes de medicina conozcan y reflexionen sobre sus propias estrategias de aprendizaje, en este trabajo se diseña e imple- menta una práctica metacognitiva en la asignatura de Fisiología Humana.

\section{Sujetos y métodos}

La práctica fue realizada como una más de las de Fisiología Humana, materia que se imparte en segundo curso de la licenciatura de medicina en la Universidad de Zaragoza. A la misma asistieron, en grupos de 10 personas, 119 estudiantes, 95 mujeres y 24 varones.

La práctica se dividió en dos partes: en la primera se puso de manifiesto que los estudiantes aprenden de distinta manera y, en la segunda, cada uno de ellos explicitó las estrategias utilizadas en el aprendizaje de la materia.

\section{Primera parte}

Para evaluar las distintas maneras de aprender de los estudiantes, se aplicó el test de Kolb [6] sobre los estilos de aprendizaje, el cual los clasifica de acuerdo con sus preferencias. La pregunta que sirve de introducción para el inicio de su elaboración, es decir, puntuación de los diferentes casilleros (cuatro) de cada línea horizontal es: ¿cómo aprende mejor?

\section{Bases metodológicas}

Desde el punto de vista teórico, este planteamiento sigue la línea de la teoría del aprendizaje experiencial de Kolb [7-9]. Como señalan Martín-García y Rodríguez-Conde [10], esta teoría ha sufrido diferentes modificaciones en las dos últimas décadas. A partir de ella, el modelo parte de la base de que para aprender algo se necesita trabajar con la información recibida que se puede obtener a partir de una experiencia directa y concreta (CE) o una experiencia abstracta (AC) -lectura, escucha-.

Las experiencias, concretas o abstractas, se transforman en conocimiento cuando se elaboran de alguna de estas dos formas: reflexionando y pensando sobre ellas (OR), o bien experimentando de forma activa con la información recibida (AE).

Kolb [7] añade que para que se produzca un aprendizaje realmente efectivo es necesario trabajar esas cuatro categorías. O lo que es lo mismo, según este modelo, un aprendizaje óptimo 


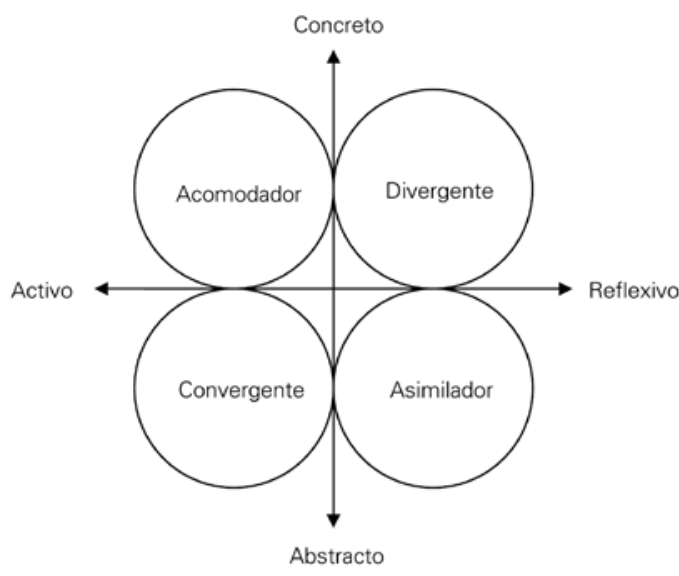

Figura 1. Estilos de aprendizaje generados a partir de la combinación entre la forma de adquirir (abscisas) y usar (ordenadas) la información. Ciclo de aprendizaje experiencial de Kolb.

es el resultado de trabajar la información en cuatro fases (Fig. 1).

En resumen, la teoría plantea dos modos de comprender (adquirir la información), mediante la experiencia directa o práctica, concreta (aprehensión), o mediante la conceptualización abstracta (comprensión) y dos modos de procesar la experiencia, mediante un proceso de extensión o exteriorización (acción) y un proceso de 'intención' o interiorización (reflexión). Estas dos raíces de los procesos cognitivos son complementarias y la base del aprendizaje real desde el más primitivo al más sofisticado. Estas cuatro categorías o procesos están enfrentados dialécticamente en dos dimensiones, como se indica en la figura precedente.

En la práctica, la mayoría de los estudiantes tiende a especializarse en una, como mucho dos, de esas cuatro fases, por lo que se puede diferenciar entre cuatro tipos de estudiantes, según la fase en la que prefieran trabajar. En función de la fase del aprendizaje en la que se especialice, el mismo contenido nos resultará más fácil (o más difícil) de aprender dependiendo de cómo se les presente y cómo lo trabajen en el aula.

\section{Instrumento}

Aunque en el Inventario de Estilos de Aprendizaje (IEA) versión III, de 1999 [8] se han superado bastantes de las debilidades psicométricas encontradas en las versiones anteriores, relativas a baja fiabilidad y consistencia interna, estabilidad y validez del constructo de la escala, en este trabajo se ha utilizado una versión anterior [6]. El cuestionario consta de nueve ítems, presenta un rango de cuatro sentencias que corresponden a los cuatro modos de aprendizaje descritos por Kolb: EC (sentir: experiencia concreta), OR (observar reflexivamente), $\mathrm{AC}$ (pensar: conceptualización abstracta), AE (hacer: experimentación activa). Cada uno de ellos se puntúa con uno de los cuatro primeros cardinales (1 a 4), y no se deben repetir las cifras. El aprendizaje individual se evalúa en dos fases: primero, se suman las puntuaciones de los cuatro modos de aprendizaje en base a una plantilla de respuestas $y$, después, se establecen las diferencias entre las puntuaciones EC-AC y ORAE que determinan el estilo de aprendizaje. Para dicha caracterización sólo se utilizan seis ítems.

\section{Segunda parte}

Se introdujo la siguiente escena: imagínese que se encuentra tomando un café con su mejor amigo/a, le recuerda que el próximo año cursará los estudios de fisiología y le suplica que le recomiende/cuente cómo estudia esta materia y qué estrategias utiliza. Se precisa que no sirven las estrategias de apoyo, tales como: ir a todas las clases, asistir a todas las prácticas, hacerse con unos buenos apuntes, propios o ajenos, comprarse un libro de texto determinado, etc. Se les proporciona un folio para que expliciten todas y cada una de las estrategias/recomendaciones utilizadas. Posteriormente, el profesor hace una pequeña introducción sobre la metacognición y los alumnos comienzan a exponer en voz alta cada una de las estrategias utilizadas, añadiéndo cada uno en su folio las que le resultan nuevas.

Esta parte se fundamenta en el diseño de una práctica previa sobre metacognición [1].

\section{Final: percepción de la actividad por parte de los estudiantes}

$\mathrm{Al}$ concluir la práctica se invita a los estudiantes a que con una frase manifiesten lo que les ha parecido la práctica.

\section{Estadística}

Se ha utilizado el sistema Excel para la recogida de datos y la estadística descriptiva se ha realiza- 
do con el sistema de aplicación informática SPSS 13.0. El nivel de significación establecido ha sido $p<0,05$.

\section{Resultados}

\section{Estilos de aprendizaje}

En la figura 2 se muestra la puntuación media obtenida por estudiantes para cada uno de los modos de aprender. Dos hechos pueden observarse en esta figura: uno, que en la conceptualización abstracta se obtiene significativamente la mayor puntación, tanto para varones como para mujeres $y$, otro, que la experimentación activa la señalan las mujeres como segunda opción (significativamente mayor que en los hombres), mientras que para los varones es la observación reflexiva.

En la figura 3 se representan los estilos de aprendizaje de los estudiantes, y es claramente manifiesta la adscripción de los mismos a los estilos asimilador (cuadro inferior derecho) y convergente (cuadro inferior izquierdo), con escasa diferencia entre ambos (52 frente a 49). Por sexos, se observa el siguiente hecho diferenciador: en los varones predominan los asimiladores (13 frente a 8), mientras que en las mujeres la mayoría es convergente (44 frente a 36 ).

\section{Metacognición}

El primer hecho a remarcar es la manifestación de la práctica totalidad de los estudiantes de que ésta era la primera vez que hablaban de las estrategias que utilizaban para aprender.

Con respecto a las estrategias utilizadas, hubo cierta unanimidad, independientemente de los estilos de aprendizaje a los que pertenecían. Ante la imposibilidad de adscribir estrategias a estilos, se comentan las señaladas por el conjunto de estudiantes:

- La utilizada más ampliamente ha sido la descongelación de la información (clarificación de puntos oscuros o no comprensibles) que sigue a la primera lectura comprensiva de los apuntes. Mayoritariamente, suelen hacerla con el libro de texto, al que sigue el diálogo con otros estudiantes, $y$ la consulta al profesor es la menos utilizada.

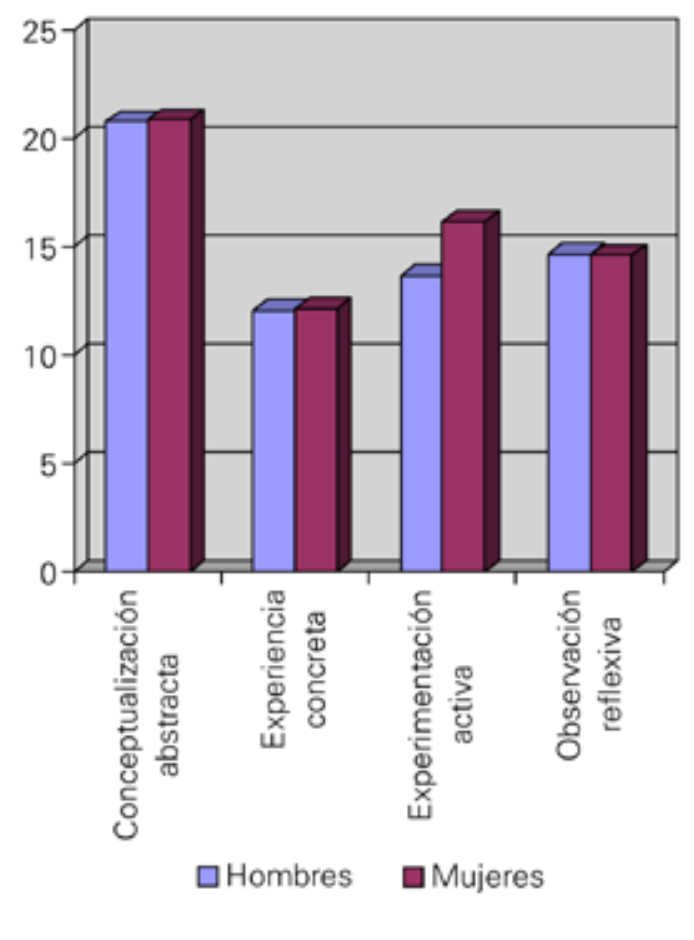

Figura 2. Orientaciones básicas del aprendizaje (modos de aprender) en estudiantes.

- También se utiliza profusamente el subrayado. Sin embargo, existe una gradación importante entre estudiantes: mientras que unos lo utilizan para señalar 'lo importante', otros lo manejan con una verdadera sofisticación, es decir, el amarillo para 'lo importante', el verde para las palabras claves, el azul claro para las novedades, el rosa para lo secundario que merece tenerse presente, etc.

- Señalan un menor uso de la utilización de los márgenes y los Post-It. Quienes así lo hacen es para recordatorios y elementos que se relacionan, establecimiento de reglas nemotécnicas y dibujos aclaradores. El dibujo también se utiliza en equivalencia con el resumen.

- Tiene una amplia aceptación la realización de resúmenes, a la que unen, en muchos casos, el establecimiento de subordinación de ideas o gradación de conceptos, algo muy próximo al mapa conceptual, que también fue indicado por un pequeño número de estudiantes. Sin embargo, esta apreciación puede ser coyuntural, ya que unos días antes se pidieron los mapas conceptuales del aparato respiratorio. Por esta razón no se recoge como estrategia aislada. 


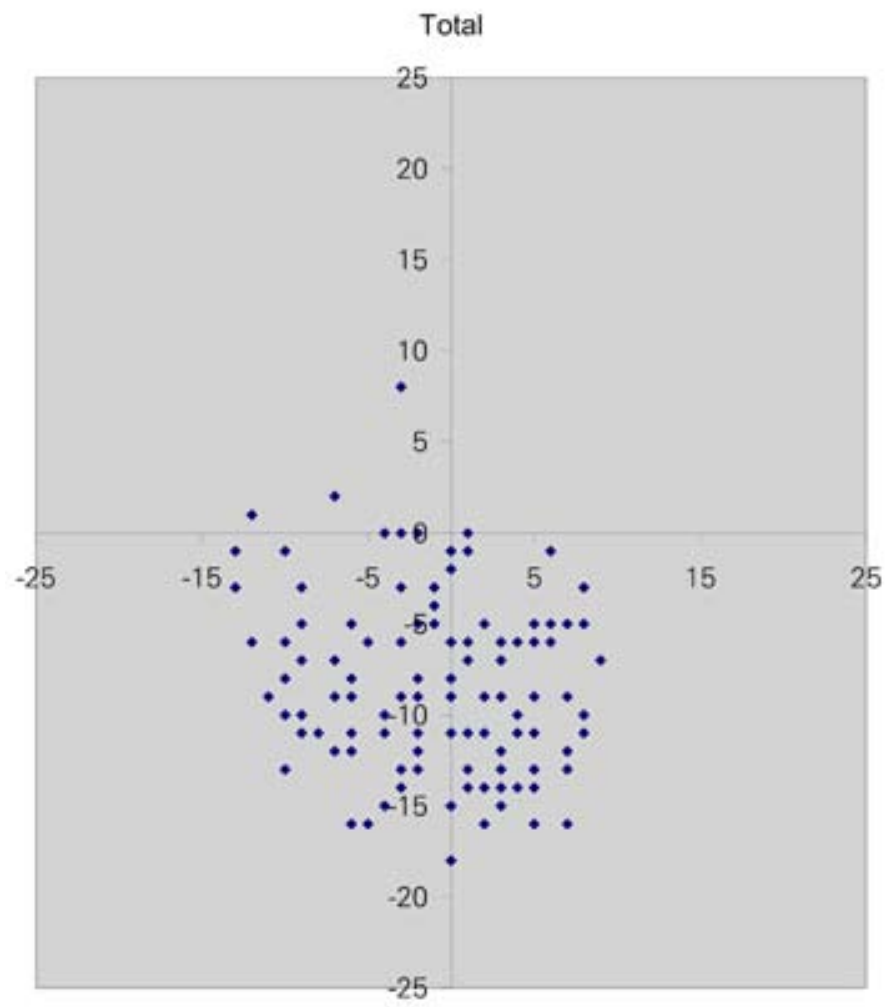

Figura 3. Estilos de aprendizaje de los estudiantes de Fisiología. Facultad de Medicina de Zaragoza. Curso 2007-2008

- Finalmente, para el repaso final, mientras que algunos/as utilizan el estudio de los resúmenes, otros/as prefieren la recitación de los temas. El mayor porcentaje los recitan en voz alta 'para sí mismos' y, en alguna ocasión, lo hacen con un familiar de primer grado a quien le cuentan el tema. Sólo ocasionalmente lo hacían entre compañeros.

Las diferencias, independientemente del estilo de aprendizaje, han consistido en la mayor o menor utilización de estrategias; algunos alumnos remarcaron la dificultad de escribirlas por ser la primera vez que lo hacían y reconocieron, después de oír a otro compañero, que ellos también la practicaban.

\section{Percepción de la práctica por los estudiantes}

La aceptación de la práctica fue unánime, a pesar de que reconocen la utilización de idénticas estrategias ('me ha parecido interesante porque he cogido alguna idea, aunque todos hacemos más o menos lo mismo'), y les resulta de gran utilidad conocer qué estrategias utilizan sus compañeros ('esta práctica ha sido muy interesante, ya que al hablar con los compañeros sobre las estrategias de estudio que utilizan te hace pensar en cómo podrías mejorar las tuyas'). A pesar de que a algunos les parecía un poco tarde hablar de este tema, porque los cambios les parecían difíciles a partir de cierta edad, una gran mayoría podrían identificarse con la siguiente trascripción, con la que se concluye este apartado: 'creo que es una práctica necesaria, que nos puede venir muy bien para el presente y el futuro' La frase más citada que resume y concluye este apartado fue: 'Muy interesante'.

\section{Discusión}

Aunque el uso metacognitivo de las estrategias supone conocerlas (saber lo que hay que hacer), 
observar, analizar y comprobar la eficacia de las puestas en juego (saber hacerlo), y saber readaptarlas, y si fuera preciso, cambiarlas, porque así lo requiere la tarea en marcha (control de la ejecución), esta práctica se ha atenido específicamente al primer apartado, informando del resto. Por otra parte y con carácter más general, se inscribe en la enseñanza reflexiva del aprendizaje y se tiene en cuenta, como plantea Pozo [11], que el aprendizaje de estrategias requiere algo más que una práctica repetitiva y reproductora de lo enseñado.

\section{Estilos de aprendizaje}

Aunque en el IEA de Kolb [8] versión III, de 1999, se han superado bastantes de las debilidades psicométricas encontradas en las versiones anteriores, en este trabajo se ha utilizado una versión anterior [6], ya que el interés perseguido consistía únicamente en poner de manifiesto las diferencias en el modo de aprender de los estudiantes, y este instrumento es el que menos tiempo requiere para su contestación.

Un instrumento que mida los estilos de aprendizaje debería utilizarse como una herramienta para animar al autodesarrollo, no sólo diagnosticando cómo la gente aprende, sino también mostrándoles cómo pueden mejorar su aprendizaje [5]. A este respecto, Garner [12] ha indicado que el autodesarrollo es más probable que resulte del incremento del conocimiento de las ventajas y debilidades relativas de los diferentes modelos por parte de los aprendedores que de la decisión de estar catalogados con la etiqueta de un particular estilo de aprendizaje.

Uno de los objetivos principales para animar a una aproximación metacognitiva se fundamenta en la incapacidad de los aprendedores para elegir la estrategia de aprendizaje más apropiada de entre una amplia variedad de opciones; sin embargo, la cuestión que permanece por responder es cómo los estilos de aprendizaje necesitan incorporarse en una aproximación metacognitiva [5]. En esta práctica se ha intentado resolver esta disyuntiva.

Por otra parte, debe insistirse que en la implementación de esta práctica se ha puesto, una vez más, de manifiesto que nuestro sistema educativo no es neutro. Si se tienen en cuenta las cuatro fases de la rueda de Kolb [6], la conceptualización es la fase más valorada, probablemente debido a que la universidad está centrada en la transmisión de conocimientos y los estudiantes traducen este hecho con una mayor puntuación y reconocimiento de este modo de aprender.

Esta constatación ha de llevar, como indica Kolb [7], a la idea de que el aprendizaje óptimo requiere de las cuatro fases, por lo que la materia se debe presentar de tal forma que se garantice la panoplia de actividades necesarias para cubrir todas las fases (modos de aprender) de la rueda de Kolb. Con esto, por una parte, se facilitará el aprendizaje de todos los estudiantes, cualesquiera que sea su estilo preferido y, por otra, se les ayudará a potenciar las fases con las que se encuentran menos cómodos.

Aunque este trabajo no pretendía catalogar a los estudiantes de Fisiología de la Facultad de Medicina de acuerdo con los estilos de aprendizaje, la práctica totalidad se hallan en los grupos convergente y asimilador, lo que coincide con una publicación previa [13], en la que se indicaba que los estilos/modos de aprendizaje de los estudiantes de medicina eran del modo converger (convergente), caracterizado por utilizar conceptos abstractos, experimentación activa y razonamientos deductivos.

En nuestro entorno, los resultados presentados en este trabajo coinciden, en su mayor parte, con los comunicados por Martín-García y Rodríguez-Conde [10], para una población universitaria de diferentes titulaciones (ciencias de la educación, estudios experimentales, humanidades, jurídico-sociales, etc.) no de medicina. En la mencionada publicación existe un predominio del grupo de los asimiladores tanto en hombres como en mujeres. Este mismo estilo, junto con los divergentes, son asimismo los que definen a otros grupos etarios [14]. En otros contextos geográficos y en nuestra titulación (Medicina), también el estilo asimilador ha sido reportado como mayoritario $[15,16]$.

\section{Metacognición}

El conocimiento de los estilos de aprendizaje puede utilizarse para incrementar la autoconciencia de los estudiantes y tutores acerca de sus fortalezas y debilidades como aprendedores [5], como se ha hecho en este estudio. Es por ello que, al concluir con la primera parte de la práctica, a cada uno de los integrantes de los dos grupos, convergentes y asimiladores, se les hacía re- 
flexionar sobre los ítems que definían los modos de aprendizaje definidores de su estilo.

Ciertamente, la práctica podría haber concluido con la primera parte, y tratar de la inclusión de los estilos de aprendizaje en la metacognición. En este caso se podría dejar que los estudiantes, tras su propio diagnóstico, opinasen sobre sus características, transfiriéndoles de esta forma toda la responsabilidad sobre el aprender y las estrategias más adecuadas [5].

En un trabajo previo [1], con respecto a este tema, se propusieron acciones metacognitivas del profesorado, del alumno y de la institución. En éste, únicamente se pretende dar unas pautas de actuación para la introducción de la enseñanza de las estrategias de aprendizaje del alumnado. Aunque muy probablemente sea en la institución $y$ entre el profesorado donde se encuentren las mayores reticencias y dificultades. En esta línea, como indican Monereo y Pozo [2], los profesores universitarios son, en gran parte, 'ex alumnos' más o menos aventajados, que en nuestro medio 'significa mayoritariamente personas competentes en anotar literalmente, retener lo copiado y transcribir lo retenido, puestos en situación de enseñar lo que les enseñó y cómo se les enseñó.

En conclusión, se ha diseñado una práctica sobre metacognición que ha sido reveladora para los estudiantes, y constituye la primera vez que han dialogado sobre este tema, con una aceptación general interesante y de interés.

\section{Bibliografía}

1. Escanero JF, Soria MS, González-Haro C. La metacognición: un camino para el éxito. II Jornadas de Innovación docente, Tecnologías de la Información y de la Comunicación e Investigación Educativa en la Universidad de Zaragoza. Zaragoza: Universidad de Zaragoza; 2008.

2. Monereo C, Pozo JI. La cultura educativa en la universidad: nuevos retos para profesores y alumnos. In Monereo C, Pozo JI, eds. La universidad ante la nueva cultura educativa. Enseñar y aprender para la autonomía. Madrid: Síntesis; 2003. p. 15-30.
3. Pérez Echeverría MP, Pozo JI, Rodríguez B. Concepciones de los estudiantes universitarios sobre el aprendizaje. In Monereo C, Pozo JI, eds. La universidad ante la nueva cultura educativa. Enseñar y aprender para la autonomía. Madrid: Síntesis; 2003. p. 33-44.

4. Fernández-Borrás J, Viscor G, Blasco J, Pagés T, Navarro I, Gallardo MA, et al. Evaluación de las preferencias y estrategias de aprendizaje de los estudiantes universitarios y su relación con la metodología docente. Aplicación a la mejora del aprendizaje. Madrid: Dirección General de Universidades; 2006.

5. Coffield F, Moseley D, Hall E, Ecclestone K. Should we be using learning styles. What research has to say to practice. London: Learning and Skills Research Centre; 2004.

6. Kolb DA. Learning style inventory technical manual. Boston: McBer; 1976.

7. Kolb DA. Experiential learning. New York: Prentice Hall; 1984.

8. Kolb DA. Learning Style Inventory version 3: technical specifications. Boston: TRG Hay/McBer; 1999.

9. Kolb DA, Boyatzis R, Mainemelis C. Experiential learning theory: previous research and new directions. In Sternberg R, Zhang L, eds. Perspectives on cognitive learning, and thinking styles. Mahwah NJ: Lawrence Erlbaum; 2001. p. 227-48.

10. Martín-García AV, Rodríguez-Conde MJ. Estilos de aprendizaje y grupos de edad: comparación de dos muestras de estudiantes jóvenes y mayores. Aula Abierta 2003; 82: 97-114.

11. Pozo JI. Aprendices y maestros. La nueva cultura del aprendizaje. Madrid: Alianza; 1998.

12. Garner I. Problems and inconsistencies with Kolb's learning styles. Educ Psychol 2000; 20: 341-8.

13. Escanero JF. Procesos psicodidácticos de la enseñanza de la medicina. Informes (ICE, Universidad de Zaragoza) 1986; 21: 77-88.

14. Martín-García AV. Estilos de aprendizaje en la vejez. Un estudio a la luz de la teoría del aprendizaje experiencial. Rev Esp Geriatr Gerontol 2003; 38: 258-65.

15. Bitran $M$, Lafuente $M$, Zúñiga $D$, Viviani $P$, Mena $B$. ¿Influyen las características psicológicas y los estilos de aprendizaje en el rendimiento académico de los estudiantes de medicina? Un estudio retrospectivo. Rev Med Chile 2004; 132: 1127-36.

16. Bitran M, Zúñiga D, Lafuente M, Viviani P, Mena B. Tipos psicológicos y estilos de aprendizaje de los estudiantes que ingresan a medicina en la Pontificia Universidad Católica de Chile. Rev Med Chile 2003; 131: 1067-78. 